\title{
Comparison of characterisation methods determining the thermal resistance of building components from on-site measurements
}

\author{
An-Heleen Deconinck ${ }^{\mathrm{a}, *}$, Staf Roels ${ }^{\mathrm{a}}$ \\ ${ }^{a}$ KU Leuven - University of Leuven, Department of Civil Engineering, Building Physics Section, Kasteelpark Arenberg 40 - bus 2447,3001 Heverlee, Belgium
}

\begin{abstract}
Reliable in-situ thermal characterisation allows to study the actual thermal performance of building components rather than the theoretical performance calculated from thermal properties of the constituent material layers. The most generally accepted method for in-situ thermal characterisation is the average method as described in ISO 9869. However, due to steady-state assumptions, the method's applicability can require long measurement periods and is often seasonally bounded. A correction for storage effects might shorten the required measurement time spans for the average method, but will not eliminate the seasonally bounded limitations. More advanced dynamic data analysis methods, such as regression modelling, ARX-modelling or stochastic grey-box modelling, can be used to overcome these difficulties. In this paper, a comparison between several semi-stationary and dynamic data analysis methods typically used for the thermal characterisation of building components from on-site measurements is made. Thereby, special attention is given to the reliability of the methods thermal resistance estimates when confronted with data sets of limited measurement time spans and different seasonal boundary conditions. First, the methods' performances are assessed for simulated measurements of a south-facing insulated cavity wall in a moderate European climate. Subsequently, the performances are examined for actual measurement data of a similar test wall.
\end{abstract}

Keywords: in-situ measurements, thermal characterisation, building component testing, average method, correction for storage effects, regression modelling, ARX-modelling, stochastic grey-box modelling

\section{Introduction}

In current thermal performance assessments of buildings, the thermal quality of a building's fabric is appraised in a theoretical way. The fabric's performance is determined from 5 the thermal properties of the constituent building components and their material layers. These properties, however, are theoretical values obtained from standards and product information. They do not account for the effects of workmanship issues, air infiltration, moisture migration, the use of different materials than the designated ones, etc. Consequently, the actual thermal performance might deviate significantly from ${ }_{35}$ the labelled one $[1,2,3,4]$. In order to assess the actual rather than the theoretical thermal quality of building components, performance labels should be based on the thermal properties 15 of building components as-built, i.e. properties estimated from on-site measurements. Hence, by relying on measured rather than theoretical values, a major source of uncertainty in the assessment of the thermal quality of building envelopes could be eliminated. Moreover, knowledge of as-built thermal properties could be used repressively to assure qualitative workmanship in the building industry.

The main challenge of characterising building components from on-site measurements is the presence of outdoor weather

\footnotetext{
${ }^{*}$ Corresponding author

Email address: AnHeleen.Deconinck@bwk.kuleuven.be (An-Heleen 50 Deconinck)
}

conditions. As the outdoor climate is intrinsically dynamic, 25 paramater such as the thermal resistance. Also, climatic conditions have a seasonally different appearance, demanding widely applicable data analysis methods. Hence, the extent to which data analysis methods are able to cope with these varying climatic conditions, will determine their practical applicability.

The most generally accepted method for the thermal characterisation of building components in-situ is the average method, described in ISO 9869 [5]. This method estimates the thermal resistance of a building component from measurements of the heat flow rate through the internal face of the component and from measurements of the (air or surface) temperatures on both sides of the component. Essentially, the average method is based on stationary boundary conditions. Yet, those are never encountered on site in practice and as a result the average method relies on averaged data as an approximation for measurements under stationary conditions. For the method to be valid, the averages should be taken over a sufficiently long period of time. This might limit the practical applicability of the method, as one usually wants as shortest measurement time spans as possible [6,7]. Next to that, the method is only valid when the heat flux through the element is negligible when compared to the change of heat storage in the component. This condition is typically hard to reach during the summer period. As a result, the application of the method is often seasonally bounded $[6,7]$. Or, in other words, the method is boundary 
condition dependent.

To improve the method's performance, the International ${ }_{110}$ Standard ISO 9869 suggests a correction for storage effects. The latter is most relevant for structures with a high R-value and high thermal mass and involves a rectification of the measured heat flow rates according to the thermal storage capacities of the element. According to the standard, this cor- -115 rection reduces the required measurement time. Nevertheless, 60 in essence, the method remains a semi-stationary analysis method with boundary condition dependencies, rather trying to cancel out the occurring dynamics inherent to the in-situ measurements than to include them in the analysis.

Yet, in contrast with these quasi-stationary methods, more 65 advanced dynamic data analysis techniques are developed to cope with the inherently dynamic character of in-situ measurements. These data analysis methods include the fluctuations of the heat flux and temperature measurements in the analysis 125 rather than to cancel them out. All methods are based on 70 inverse modelling techniques, meaning that data-driven models are constructed from the available measurement data of the studied building component. The estimated models then reveal information on the thermal performance of the studied building ${ }_{130}$ component.

75 In the context of in-situ thermal characterisation, one of the first dynamic methods to analyse heat flux and temperature measurements was proposed by $[8,9,10,11]$. Their suggested approach models the heat flux through the studied building ${ }_{135}$ component by a stationary and a transient part. This transient

80 part is aimed at covering the influences of the varying climatic conditions exciting the building component, so that the steadystate behaviour can be isolated in the stationary part. By fitting this model to the heat flux measurements using a multiple linear ${ }_{140}$ regression, the thermal conductance of the studied building

85 element can be estimated. In essence, the stationary part of the model is linked to the momentary temperature differences over the component and thus to the thermal conductance, while the transient part takes into account several time constants ${ }_{145}$ connected to in- and external temperatures changes in the past

90 at both sides of the component. This dynamic analysis method is described in Annex B of ISO 9869. Also, a very similar approach, based on the same principles but excluding the use of time constants is described in $[12,13]$.

Further evolution in dynamic data analysis of in-situ mea95 surements led to the use of more general AR(MA)X-models $[14,15]$. The latter also resurfaced more recently in the context of the thermal characterisation of building components $[16,17]$. ARX-models are completely black-box, meaning ${ }_{155}$ that the model parameters have no direct physical meaning. In

100 essence, ARX-models describing the heat transfer in building components model the heat flux at a certain moment in time in function of the measured temperatures at that moment and in function of measured temperatures and heat fluxes in the past. By means of multiple linear regression, these ARX-models can

be fitted to the heat flux measurements. Although the estimated ${ }_{160}$ model parameters have no direct physical significance, the gain of the model gives information on the stationary behaviour of the system and thus on the thermal resistance of the studied element.

Along with the renewed use of ARX-models, the possibilities of stochastic state space modelling for thermal characterisation purposes are studied [18, 19, 20, 21, 22, 23, 24]. In contrast with the previous model class, these models are grey-box, meaning that the model formulation is based on prior physical knowledge and that a direct physical interpretation can be attached to the model parameters. Essentially, stochastic grey-box models are lumped resistance-capacitance models formulated in a stochastic state space form. The models describe the heat flux in function of thermal resistances, capacitances and temperatures in the wall. The model parameters are typically estimated by a maximum likelihood approach in a one-step prediction setting $[25,26]$. The estimated resistances and capacitances then directly provide the required thermal information on the studied building component.

The referred literature proves that the subject of data analysis methods for in-situ thermal resistance characterisation of building components has already widely been studied. In some studies, the performances of the available methods have been mutually compared or the performances have been tested against different climatic conditions and different measurement time spans [7, 11, 21, 23, 24, 27]. However, as far as known by the authors, a large-scale comparison of all techniques' performances in function of the measurement time span and climatic conditions is absent. In this paper, such a comparison is made. The performances of more common semi-stationary methods are compared with the performances of more advanced dynamical data analysis methods for the thermal characterisation of a south-facing insulated cavity wall. This type of wall is chosen on purpose because of the two capacitive layers on both sides of the insulation hindering the thermal characterisation procedures. In the comparative assessment, special attention is given to the reliability of the methods' estimation results when confronted with data sets of limited measurement time spans and different seasonal boundary conditions. As such, the boundary condition dependency of the different methods is studied. In a first part, the performances of all characterisation methods are systematically compared for simulated measurement data of a south-faced insulated cavity wall in a moderate European climate. The use of simulated data allows for an exact evaluation of the estimated values compared to the 'real' thermal resistance of the studied wall. Furthermore, the use of simulated data allows to focus on the methods' ideal performance excluding all measurement errors possibly jeopardizing a proper thermal resistance estimation. In a next step, the performances of the different techniques are tested for real measurement data of a south-facing cavity wall.

\section{Methodology}

In order to evaluate and compare the performance of the different analysis techniques, all methods are applied on various data sets of a south-facing insulated cavity wall. The same outputs and inputs are used for all methods and will be limited to the typically used measurements for in-situ characterisation, 
i.e. the internal heat flux and the in- and external surface tem-210

peratures of the observed wall. In order to compare the models' performance, several data sets with different lengths and in different seasons are selected.

\subsection{Case study}

At this stage, the heat flux and temperature 'measurements' are obtained from simulations of a cavity wall in a typical Belgian climate. The wall is simulated with HAMFEM, a finite element program based on the standard partial differential equations of heat, air and moisture transfer in porous building materials [28]. The thermal properties used for the onedimensional simulations with HAMFEM are represented in table 1 and the goal value for the total thermal resistance of the cavity wall adds up to $4.002 \mathrm{~m}^{2} \mathrm{KW}^{-1}$. Constant thermal properties are assumed for the simulations to exclude temperature and moisture dependencies of the wall's thermal resistance. The wall is simulated with a fine mesh of 200 elements and 201 nodes. In order to mimic realistic measurement results, both system and measurement noise are added to the simulated data. The assumption of white noise is made for both noise types. The surface temperatures and internal heat flux that ${ }^{230}$ result from this HAMFEM-simulation will serve as the data analysis methods' inputs and output respectively.

A simulation with the length of one year and a calculation time step of one minute is performed for the typical moderate climate of Uccle (Belgium). Irradiance and outdoor air tem- ${ }^{23}$ perature data with a time resolution of 1 minute are obtained by Meteonorm v6.1 based on the period of 1981-2000. Other climate data is obtained with a time resolution of 1 hour and is interpolated to minutely data. For the exterior heat balance, the convective heat transfer coefficient is assumed to be function of the wind velocity according to [29]. For the calculation of short and long wave radiation, an absorption coefficient $\alpha_{S}$ of 0.5 and an emissivity $\epsilon_{L}$ of 0.9 is assumed for the brick façade. This corresponds to a brick with a rather light colour. For the indoor boundary conditions, two scenario's are regarded. In first scenario, a constant indoor air temperature of $20^{\circ} \mathrm{C}$ is maintained during the whole year, although in summer no cooling is applied. Hence, during warmer periods, the indoor air temperature can exceed the set temperature of $20^{\circ} \mathrm{C}$ and result in periods with a free floating indoor air temperature. the second scenario, no heating nor cooling is applied during summer which results in a full-time free floating indoor air temperature during the summer months. To simulate the free-floating indoor air temperature, a single zone adjacent to the wall is included in the HAMFEM-simulations. Heat

Table 1: Thermal properties of the simulated cavity wall from outside to inside.

\begin{tabular}{llll}
\hline & $\mathrm{d}$ & $\mathrm{R}$ & $\mathrm{C}$ \\
& {$[m]$} & {$\left[m^{2} K W^{-1}\right]$} & {$\left[M J m^{-2} K^{-1}\right]$} \\
\hline brick & 0.090 & 0.113 & 0.357 \\
insulation & 0.127 & 3.614 & 0.007 \\
brick & 0.140 & 0.187 & 0.236 \\
plaster & 0.015 & 0.088 & 0.018 \\
\hline TOTAL & $\mathbf{0 . 3 7 2}$ & $\mathbf{4 . 0 0 2}$ & $\mathbf{0 . 6 1 8}$
\end{tabular}

transfer from wall to zone is modelled by a constant interior heat transfer coefficient of $8 \mathrm{Wm}^{-2} \mathrm{~K}^{-1}$. Furthermore, the zone is ventilated with an air change rate of 0.5 . Therefore, a room with a ground surface of $30 \mathrm{~m}^{2}$ is considered. The heigt of the room measures $2.8 \mathrm{~m}$ and the room has two exterior and 5 two interior walls. Room heating is only applied in the first scenario when the temperature drops below the set temperature of $20^{\circ} \mathrm{C}$.

\subsection{Data sets}

To compare the different analysis methods' performances, various data sets are selected for analysis purpose: (1) data sets with different lengths and (2) data sets in different seasons. Three main seasonal periods of 60 days are considered: winter, spring and summer. These main periods are denoted by the month these periods start in: January, April and July respectively. As previously explained, the summer period will appear twice: once with a semi-controlled indoor air temperature at $20^{\circ} \mathrm{C}$ or higher (denoted July) and once with a full-time free floating indoor air temperature (denoted Julyfree). These four main periods are represented column wise in figure 1. From those main periods, diverse data sets are constructed: data sets with lengths ranging from 1 to 30 days, and data sets starting from the 1st of January, April and July till the 30th of these months. In fact, the different data sets are constructed by using a moving window advancing with a one day step and repeated for different window lengths. Each of these data sets will result in a thermal resistance estimate for the studied cavity wall for all different methods. The ensemble of the results will allow to study the accuracy of the different methods in function of the measurement time span and the measurement period throughout the year.

\section{Characterisation methods}

\subsection{Average method}

The most generally accepted method for the thermal characterisation of building components on site is the average method as formulated in ISO 9869 [5]. This method is based on the definition of the thermal resistance of a building element equalling the temperature difference over the element divided by the heat flow rate going through the element under steady-state conditions. However, since stationary boundary conditions are never encountered on-site, the average method relies on averaged data as an approximation for measurements under stationary conditions. Hence, the thermal resistance is calculated as

$$
R=\frac{\sum_{j=1}^{n}\left(T_{s i, j}-T_{s e, j}\right)}{\sum_{j=1}^{n} q_{j}}
$$

with $R$ the total thermal resistance of the element $\left(m^{2} K W^{-1}\right)$, $T_{s i, j}$ the internal surface temperature at reading $j\left({ }^{\circ} \mathrm{C}\right), T_{s e, j}$ the external surface temperature at reading $j\left({ }^{\circ} \mathrm{C}\right), q_{j}$ the internal 

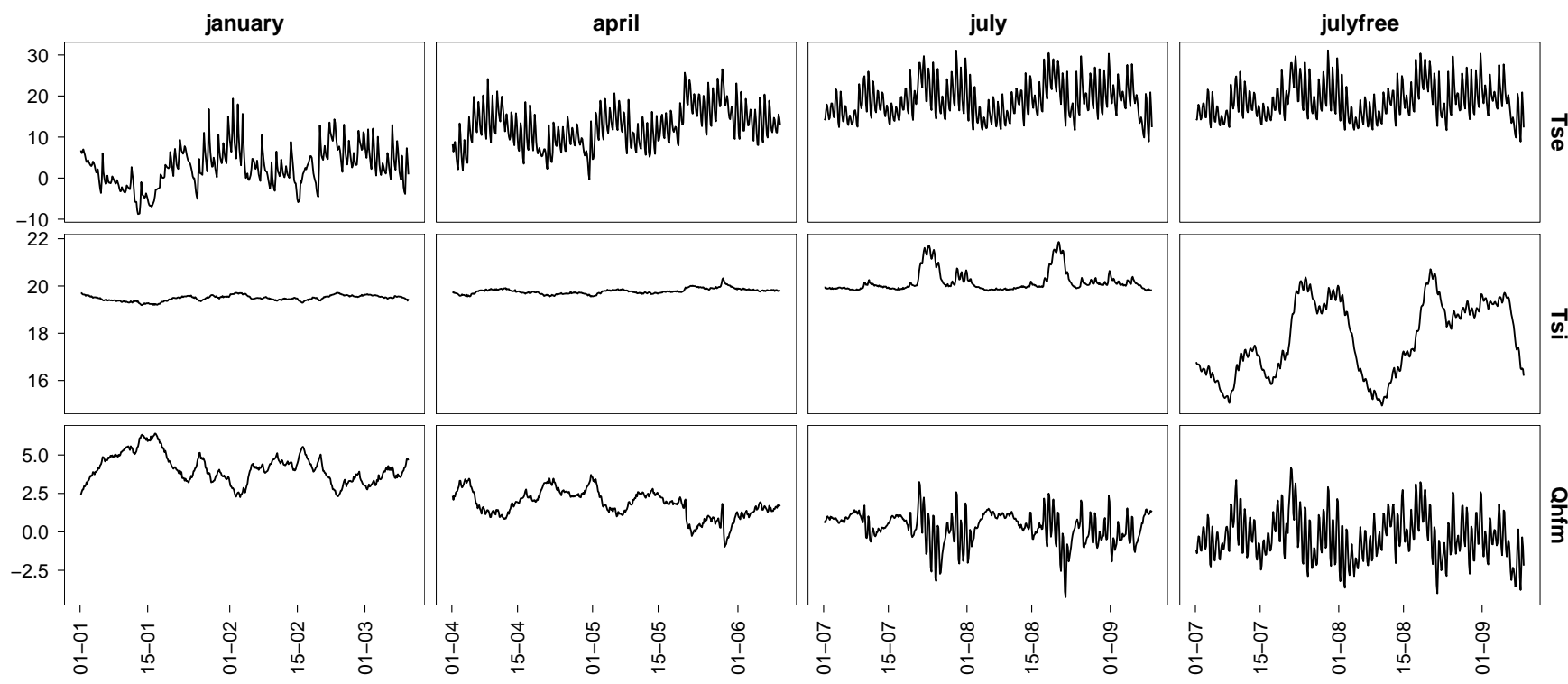

Figure 1: Simulated measurement data for the different seasonal periods with $T_{s e}$ the external surface temperature $\left({ }^{\circ} \mathrm{C}\right), T_{s i}$ the internal surface temperature $\left({ }^{\circ} \mathrm{C}\right)$ and $Q_{h f m}$ the heat flow rate $\left(W \cdot m^{-2}\right)$.

heat flow rate at reading $j\left(W^{-2}\right)$ and $n$ the number of mea-290 sured data points (-).

The assumption of averaged data equalling steady state data is only valid under certain conditions. First, the thermal properties of the component's materials should be constant over the range of temperature fluctuations occurring during the test. In reality, the thermal conductivity is rarely constant, but is often temperature and moisture dependent. However, for the simula- ${ }^{295}$ ion research, these influences are omitted and constant thermal properties are assumed. Another validity condition requires that the change of amount of heat stored in the element should be negligible when compared to the amount of heat going through the element. This condition is typically hard to reach during ${ }^{300}$ ummer periods due to the increased capacitive working of a building's fabric. Hence, the method's practical applicability is often seasonally bounded.

More generally, in order to obtain reliable estimation results, the averages should be taken over a sufficiently long period of ${ }^{30}$ time so that the dynamic behaviour of the building component can be cancelled out. This might limit the applicability of the method, as one usually wants as shortest measurement time spans as possible. A guidance of the measurement durations is provided by ISO 9869. The norm formulates criteria determining, during the course of the measurements, when sufficient data has been recorded to obtain reliable results. These criteria are based on the magnitude of the deviations between the subsequently obtained R-estimates computed after each measure-- ${ }^{310}$ ment. So, essentially, the criteria assess the convergence of the estimator. Nevertheless, no additional information on the accuracy of the obtained thermal resistance estimate is provided.

According to ISO 9869, the recording measuring interval is typically $0.5 \mathrm{~h}$ to $1 \mathrm{~h}$. In this paper, however, the average method ${ }^{315}$ is applied on four hour averaged data. As averages are the ba- sis of the average method, this does not affect the estimation results.

\subsection{Average method with correction for storage effects}

Based on steady-state assumptions, the average method supposes that all the heat flux measured at the interior surface of the building component passes through the test element. Strictly speaking, this is only the case if the amount of heat stored in the element is the same at the start and at the end of the test. In reality, this is often not the case, especially for structures of high R-value and high thermal mass. Therefore, ISO 9869 [5] suggests a correction for storage effects to the average method. The latter involves a rectification of the heat flow rates according to the thermal storage capacities of the element. The following equation represents these adjustments to the measured heat flux at each data point, involving internal and external thermal mass factors for the structure concerned.

$$
\sum_{j=1}^{n} q_{j}-\frac{\left(F_{i} \delta T_{i}+F_{e} \delta T_{e}\right)}{\Delta t}
$$

with $F_{i / e}$ the internal/external thermal mass factors relying on reasonable estimates of the thermal mass and resistance of the various layers of the structure (-) [5], $\delta T_{i}$ the difference between the internal temperature averaged over the $24 \mathrm{~h}$ prior to reading $j$ and the internal temperature averaged over the first $24 \mathrm{~h}$ of the analysis period $(K), \delta T_{e}$ the difference between the external temperature averaged over the $24 \mathrm{~h}$ prior to reading $j$ and the external temperature averaged over the first $24 \mathrm{~h}$ of the analysis period $(K)$ and $\Delta t$ the time interval between two readings $(s)$. Note that the calculation of the thermal mass factors requires prior knowledge or good assumptions about the thermal mass and resistance of the various layers of the 

components from on-site measurements. Energy and Buildings 130: 309-320. doi.org/10.1016/j.enbuild.2016.08.061

structure.

In this paper, four hourly data is used for the data analyses by this method. According to the standard, the correction often permits a shorter measurement time. However, no indication on the obtained accuracy of the estimated parameters is provided by the method.

\subsubsection{Anderlind's regression method}

In the context of in-situ thermal characterisation, one of the first dynamic methods to analyse heat flux and temperature measurements was proposed by $[8,9,10,11]$. This method is also adopted in Annex B of ISO 9869. A very similar, but more direct approach of this dynamic method was developed by ${ }^{380}$ Anderlind [12,13] and will be applied in this paper. In Anderlind's method the variations of the heat flux through a building component are modelled by three different parts: a first part including the stationary behaviour of the heat flux measurements and a second and third part describing the momentary fluctua- ${ }^{385}$ tions of the heat flux as a response to temperature changes of the indoor and outdoor surface temperatures in the past, as can be seen in

$q_{j}=\frac{1}{R}\left(T_{s i, j}-T_{s e, j}\right)+\sum_{l=j-p}^{j-1} A_{l}\left(T_{s i, l+1}-T_{s i, l}\right)+\sum_{l=j-p}^{j-1} B_{l}\left(T_{s e, l+1}-T_{s e, l}\right)$

with $T_{s i}$ the internal surface temperature, $T_{s e}$ the external ${ }_{340}$ surface temperature, $R, A_{l}$ and $B_{l}$ the regression coefficients and $p$ the number of historical data points that are used.

The coefficients $R, A_{l}$ and $B_{l}$ are estimated by a multiple lin-395 ear regression. The method relies on the fact that the transient art covers the variations in the heat flux measurements induced by the varying climatic conditions, so that the steady-state behaviour of the wall can be isolated in the stationary part. In this way, the estimated coefficient of the momentary tempera-400 ture differences can be interpreted as the thermal conductance, or the inverse of the thermal resistance. Note that the model assumes a zero intercept and that, in that case, $R^{2}$-values are no reliable indicators of goodness of fit. These values must therefore be carefully interpreted. Also, a standard deviation of the 405 estimated conductance is estimated by the multiple linear regression calculations. Hence, an indication on the accuracy of the estimated parameter is provided. However, again, this value must be carefully interpreted because, in essence, it evaluates the parameter's fit rather than its physical interpretation.

Application of Anderlind's regression method is very 360 straight-forward. The only choice that has to be made is how far the method will look back in time. Or, in other words, how many temperature differences from the past will be included in the model. In this paper, models are applied that include data 415 back in time during one sixth of the total measurement length with a maximum of three days. For example, a data set of 12 days will include temperature differences up till two days back in time. Consequently, only ten days will be used to fit the model on. A data set of 30 days, for example, will include ${ }_{420}$ temperature differences up till three days of data points from the past. The remaining 27 days will be used to fit the model on. Measurement data with a sample time of four hours will be used.

\subsubsection{ARX-modelling}

ARX-models are another class of dynamic models used to retrieve the thermal resistance of building components from onsite measurements. In essence, these models are data-driven models constructed to mimic the input-output behaviour of the observed system. The parameters that are thereby estimated are basically scaling factors to adjust the model output to the measured output [30]. The model structure is very general so that the estimated parameters have no direct link to the internal physical functioning of the system. Nevertheless, the models are able to estimate the thermal resistance from building component measurements for as the gain of such models reveals information on the stationary behaviour of the studied element.

ARX-models used for thermal resistance characterisation describe the heat flux through a building component at a certain moment in time as a function of the measured surface temperatures at that moment in time and of measured surface temperatures and heat fluxes in the past. By means of multiple linear regression, the modelled heat fluxes are then fitted to the measured data. Such ARX-models are formulated as

$$
Q(B) q_{j}=\omega_{s i}(B) T_{s i, j}+\omega_{s e}(B) T_{s e, j}+e_{j}
$$

with $Q(B)=1+Q_{1} B^{1}+\ldots+Q_{n q} B^{n q}$,

$\omega_{s i}(B)=\omega_{s i, 0}+\omega_{s i, 1} B^{1}+\ldots+\omega_{s i, n i} B^{n i}$

$\omega_{s e}(B)=\omega_{s e, 0}+\omega_{s e, 1} B^{1}+\ldots+\omega_{s e, n e} B^{n e}$

Hereby, $B$ is the back shift operator, $e_{j}$ the simulation error, $Q(B)$ the input polynomial and $\omega_{s i}(B)$ and $\omega_{s e}(B)$ the output polynomials. The polynomials are each characterised by their order: $n q$ is the order of the output polynomial and $n i$ and $n e$ are the orders of the input polynomials related to the indoor and outdoor surface temperatures respectively. The polynomial orders basically indicate how many data points from the past are involved to explain the heat flux at reading $j$.

The stationary behaviour of an ARX-model, i.e. the gain of the model, can be obtained by setting the back shift operator to $1[16,31]$. Generally, a model's gain is the steady-state reaction of the model output to a model input. Based on a comparison of the ARX-model's equation 4 with the general equation for steady-state heat transfer $q=\frac{1}{R}\left(T_{s i}-T_{s e}\right)=H\left(T_{s i}-T_{s e}\right)$, two stationary parameters can be calculated: one thermal conductance $H_{i}$ related to the indoor surface temperature $T_{s i}$ and one thermal conductance $H_{e}$ related to the outdoor surface temperature $T_{s e}$ (equation 5). Based on a minimum variance weighting (equation 6), the two estimates can be combined into a total thermal conductance estimate [16, 31]. The estimation result is accompanied by a standard deviation giving information on the accuracy of the estimated parameter. 


$$
\begin{gathered}
H_{i}=\frac{1}{R_{i}}=\frac{\omega_{s i}(1)}{Q(1)} \quad ; \quad H_{e}=\frac{1}{R_{e}}=\frac{-\omega_{s e}(1)}{Q(1)} \\
H_{\text {tot }}=\frac{1}{R_{t o t}}=\lambda H_{i}+(1-\lambda) H_{e}
\end{gathered}
$$

with $\lambda$ the value that minimizes the variance of $H_{t o t}$.

The use of ARX-modelling requires two choises: (1) a proper selection of the polynomial orders and (2) a selection of a feasible sampling time. Both choices must be made in function of the use of the model. In the case of thermal resistance characterisation, the focus is entirely on estimating a stationary parameter. Hence, the higher frequency spectrum corresponding to faster dynamics is of minor importance. Therefore, averagin the measurement data, which is a low pass filter, is advised [31]. In this paper, four hourly averaged measurement data is used.

Concerning the polynomial order selection, a stepwise backward model selection procedure based on the Akaike Information Criteria (AIC) is applied. This procedure typically fits a maximum order model and looks for the term whose removal has the most positive impact on the AIC. After elimination of this term, a simplified model is obtained. This process will be repeated till further stepwise removal of terms will no longer improve the AIC. Analogous to the application of Anderlind's method, in this paper, the maximum order model specified at the beginning of the ARX-model selection procedure corresponds to a model including data back in time during one sixth of the total measurement length with a maximum of three days.

ARX-modelling results in thermal resistance estimates accompanied by a standard deviation giving information on the obtained accuracy of the estimate. Next to that, ARXmodelling also allows to estimate the time constants of the studied wall.

\subsubsection{Stochastic state space modelling}

A third class of models to dynamically analyse in-situ measurements of building components are stochastic grey-box models. The use of grey-box models is an approved method for identifying systems in a lot of domains and has more re-480 cently been studied in the context of thermal resistance characterisation of building components [18, 19, 20, 21, 22, 23, 24]. The method constructs physical data-driven models of building components by tuning the behaviour of these models to the measured heat fluxes through the components. The assumed ${ }_{485}$ physical models describe the heat transfer in the studied elements as a set of continuous stochastic differential equations formulated in a state space form. The model parameters that are thereby used, and that scale the model output to the measurements, have a direct physical significance. Basically, these ${ }_{490}$ models are lumped parameter models consisting of thermal resistances and capacitances.

The state space model structure that is used in this paper for modelling the insulated cavity wall is derived from the resistance capacitance model represented in figure 2 . A third-order ${ }_{495}$ model is considered, meaning that the thermal mass of the wall

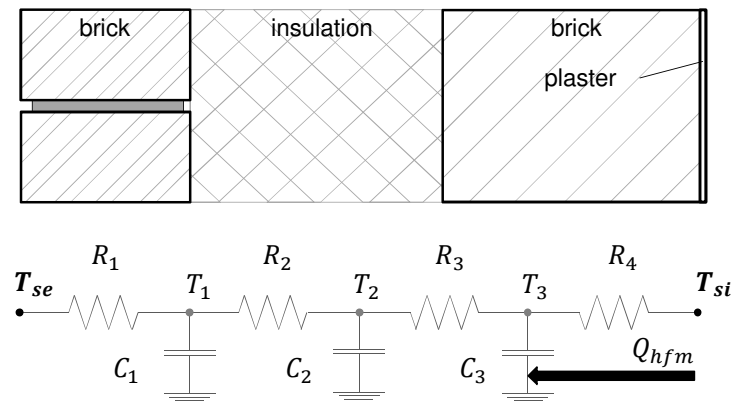

Figure 2: A third order model structure for building components represented by its RC-network. Note that the location of the temperature nodes in this figure is arbitrarily chosen and that they are not necessarily equally distributed over the wall. The identification procedure determines the values of the model resistances and controls the location of the capacities in the modelled wall. Hence, the estimated model resistances and capacitances do not necessarily correspond to the resistances and capacitances of the different wall layers.

is lumped into three capacitances, and described by the equations

$$
\begin{aligned}
& d T_{1}=\frac{1}{C_{1} R_{1}}\left(T_{s e}-T_{1}\right) d t+\frac{1}{C_{1} R_{2}}\left(T_{2}-T_{1}\right) d t+\sigma_{1} d \omega_{1} \\
& d T_{2}=\frac{1}{C_{2} R_{2}}\left(T_{1}-T_{2}\right) d t+\frac{1}{C_{2} R_{3}}\left(T_{3}-T_{2}\right) d t+\sigma_{2} d \omega_{2} \\
& d T_{3}=\frac{1}{C_{3} R_{3}}\left(T_{2}-T_{3}\right) d t+\frac{1}{C_{3} R_{4}}\left(T_{s i}-T_{3}\right) d t+\sigma_{3} d \omega_{3} \\
& q_{k}=\frac{1}{R_{4}}\left(T_{s i, k}-T_{3, k}\right)+\epsilon_{k}
\end{aligned}
$$

with $R_{i=1, \ldots, 4}$ the model resistances, $C_{i=1, \ldots, 3}$ the model capacitances, $T_{i=1, \ldots, 3}$ the state variables representing the temperatures of the thermal capacitances, $t$ the time, $\omega_{i=1, \ldots, 3}$ the standard Wiener processes and $\sigma_{i=1, \ldots, 3}$ the incremental variances of the Wiener processes. The surface temperatures $T_{s e}$ and $T_{s i}$ are the inputs of the model. The internal heat flux $q_{k}$ is the observed variable with $k$ indicating the discrete time point of the measurements and with $\epsilon_{k}$ the measurement error which is assumed to be a Gaussian white noise process with variance $\sigma^{2}$. The total wall resistance is determined as the sum of the different model resistances.

In this paper, the models' parameters are estimated using the Continuous Time Stochastic Modelling (CTSM-R) toolbox implemented in the statistical software R (http://ctsm.info/). CTSM-R uses maximum likelihood estimation to identify the unknown parameters for the given model structure in a one-step prediction setting. This particular way of inverse modelling allows the use of statistical tools for validation of the estimated models [18].

Grey-box modelling provides parameter estimates with a standard deviation indicating the accuracy by which the parameters are estimated. Furthermore, in contrast to the other dynamic analysis methods, not only the thermal resistance of the 
wall can be estimated, but also the effective thermal capacity of the wall and the dominant time constants. Again, measurement data with a sampling time of four hours is used.

\section{Results}

The thermal resistance estimates resulting from the different analysis methods for all data sets and all data periods are summarised in figure 3. Each data point in the figure corresponds 560 to an R-estimate of a different data set. The results are plotted

505 in function of the length of the data sets, i.e. the number of days included in the analysed measurement data. Note that the boundaries of the $y$-axis are adjusted for the results of the semi-stationary methods in January and April and that some ${ }_{565}$ data points corresponding to the other methods or to summer periods fall outside the boundaries of the graph. The reference value for the thermal resistance of the cavity wall is indicated in the graph as the red dotted line. The grey dotted lines represent the $5 \%$ and $10 \%$ accuracy bands around the goal value. The 570 figure is constructed as follows: the results for the different data periods throughout the year are ordered column wise, while the results for the different analysis methods are ordered row wise, with a repetition of the results for the average method in grey as a reference to the other models' results.

Examining the results of the average method, it can be seen from the top row of figure 3 that the R-estimates for January converge to the goal value when long enough data periods are considered. For short data sets, the spread on the results is rather large. Only for data sets of around 8 days580 or longer, all results lie within a $10 \%$ accuracy band around the goal value. Data periods of around 20 days or longer are required to obtain 5\% accurate results in January. In April, similar results are observed, although even longer data sets are required to obtain the same accuracies on the R-estimates.585 Due to the increased dynamic excitation of the cavity wall, around 12 to 14 days or longer are needed to obtain results in between the $10 \%$ accuracy bands. Also, no long enough data periods were examined resulting in all R-estimates within a 5\% accuracy band. Furthermore, the R-estimates for the tw0590 summer scenarios in July clearly show the limited validity of resistance are acquired because both summer periods are characterised by small heat flow rates and a large capacitive functioning of the wall.

If the correction for storage effects is applied on the data

540 (second row of figure 3), an improvement of the estimation results in January and April is found compared to the average method. In January, data sets of around 3 to 4 days already lie within a $10 \%$ accuracy band and data sets of 6 days in 600 a 5\% accuracy band around the goal value. In April, data sets containing around 8 and 14 days are required in order to obtain $10 \%$ and $5 \%$ accuracy levels. Hence, the correction for storage effects improves the results for winter and spring measurements. Note, however, that the correction in this paper 605 is applied in an optimal way: the thermal properties that are required for the calculation of the thermal mass factors are exactly known for the studied wall. In reality, accurate thermal properties will rarely be known and hence the improvement of the correction will be less effective. Besides, the results for the two summer scenarios in July show that the correction is not able to improve the results for typical summer measurements that are characterized by small heat fluxes fluctuating around zero and an active heat storage.

Underneath the results for the semi-stationary methods, the results of the dynamic data analysis methods are represented in figure 3. It is shown that in case of Anderlind's regression method a fast and accurate convergence of the R-estimates to the goal value is achieved in January and April. Data sets of 5 and 6 days are sufficient to obtain results with accuracies higher than 5\% in January and April respectively. Anderlind's regression model also converges to the reference value during the two summer scenarios. Data periods of around 11 and 22 days are required for $10 \%$ and $5 \%$ accurate results for both scenario's in July. Although, generally, the scenario with a full-time free floating indoor air temperature (Julyfree) has a wider spread on the results than the scenario with a semi-controlled indoor air temperature (July).

The estimates deduced from the ARX-models in figure 3 show similar results compared to estimates from Anderlind's method. In January and April, small data sets from around 5 and 6 days contain sufficient information for highly accurate estimation results of the wall's thermal resistance. Also during both summer periods, accurate results are obtained, however, compared to the estimates from Anderlind's models, more outliers are observed. Nevertheless, the method's estimated standard deviations do indicate the less reliable results, as can be seen from figure 4 . The graph shows that the results which deviate more from the reference value are characterised by larger estimated standard deviations.

Finally, the R-estimates resulting from the GREY-box models are also represented in figure 3. From this figure, it can be seen that data sets containing one or two days have no estimation results. This is due to the fact that those data sets contain too little data points for the analysis method to be operational.

In January and April, again very accurate estimation results can be obtained in short data periods. Around 4 and 5 day periods already allow to estimate the thermal resistance of the wall with 5\% accuracy. For the summer scenarios in July, as for the other methods, longer data periods are required to obtain accurate results. Thereby, it seems that more difficulties are encountered for the scenario with a semi-controlled indoor air temperature. In those situations, data periods of at least 18 days are required in order to obtain $5 \%$ accurate results. In the scenario with a full-time free floating temperature, grey-box models perform very well, also compared to the other methods, and reach results within $10 \%$ and 5\% accuracy bands in 11 and 20 days respectively. Again, less reliable results are indicated by their estimated standard deviations, as can be seen from figure 4 .

In addition to figure 3 , the most important results are also numerically summarized in table 2 for data sets containing one week ( 7 days) and one month (30 days) for the scenarios january and julyfree. For each of these subsets, the table represents the mean estimated thermal resistance and standard deviation 


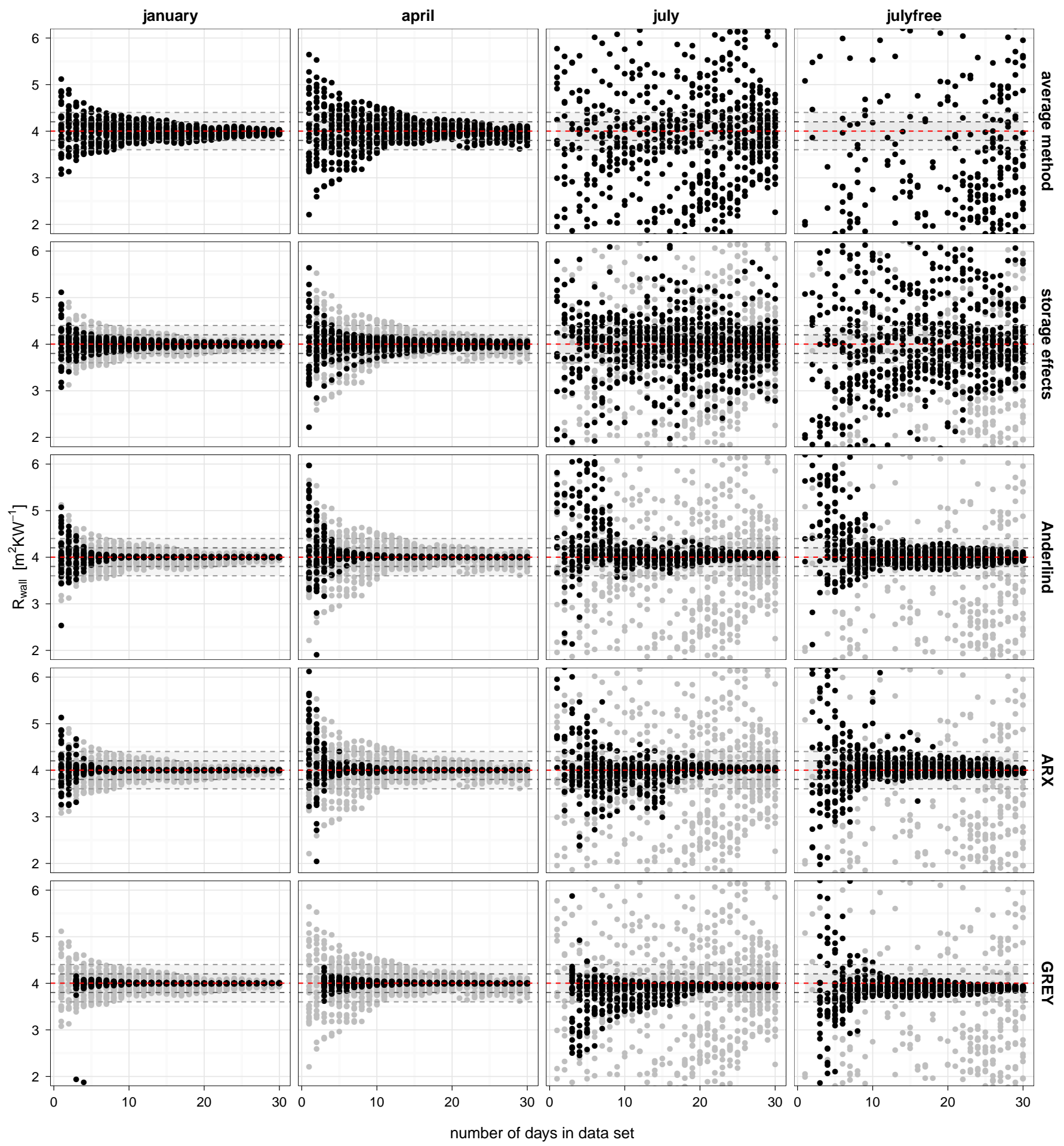

Figure 3: Comparison of the different analysis techniques regarding the data set length and period. The red dotted line represents the reference value for the thermal resistance of the cavity wall. The grey dotted lines and areas correspond to the $5 \%$ and $10 \%$ accuracy bands.

together with the minimum and maximum estimated thermal mean, minimum and maximum estimated thermal resistance and the theoretical goal value are represented in grey.

For a general comparison between the analysis methods, fig-

ure 5 represents the spread on the estimation results for the different methods as a function of the length of the data sets. The spread is calculated as the standard deviation of the obtained estimation results per data set length and for each period and analysis method. Figure 5 clearly shows the advantages of the 
Table 2: Summary of the estimation results for data sets containing one week ( 7 days) and one month (30 days) of data for the scenarios january and julyfree. For each of these subsets, this table represents the mean estimated thermal resistance (average) and standard deviation (sd) together with the minimum (min) and maximum $(\max )$ estimated thermal resistance. In grey italic, the absolute difference between the mean, minimum and maximum estimated thermal resistance and the theoretical goal value are represented. All values have the unit of $\left(m^{2} K W^{-1}\right)$.

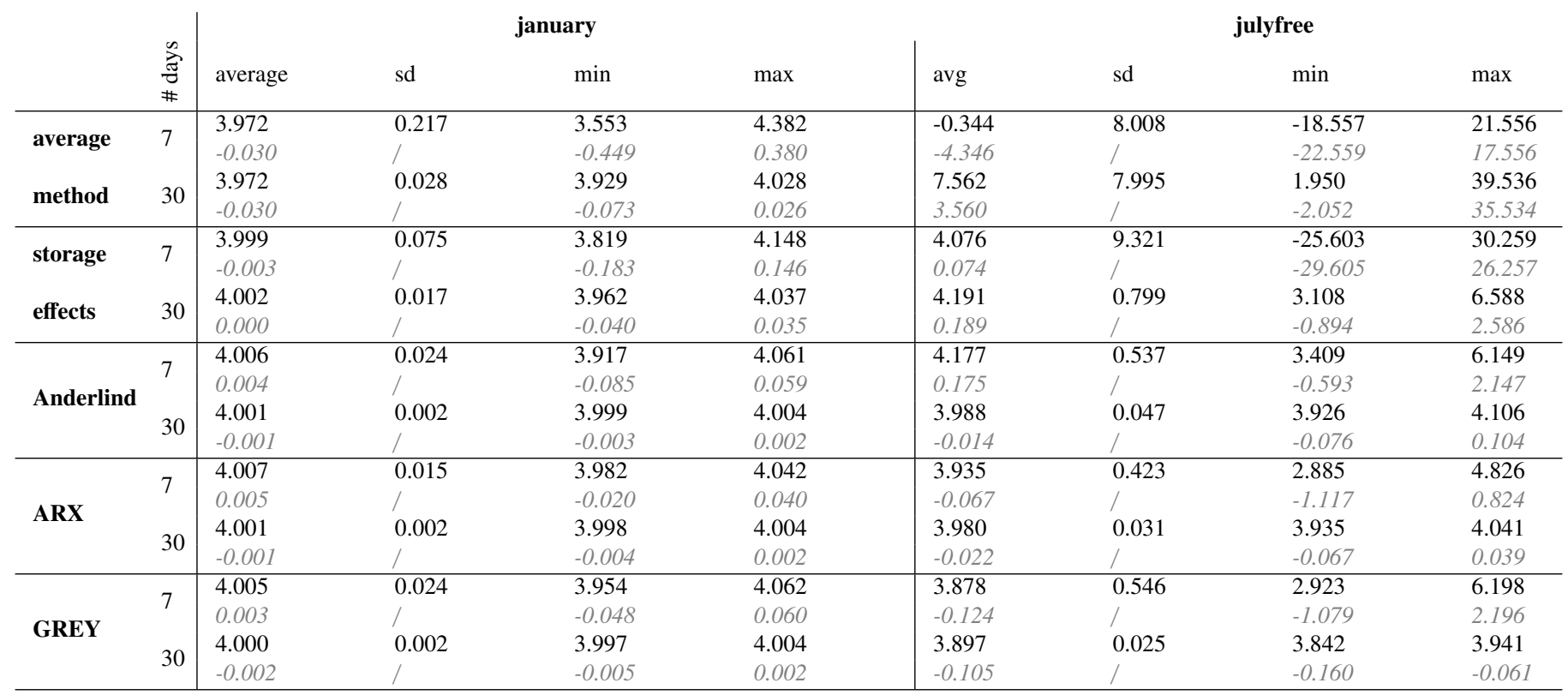

dynamic data analysis methods compared to the semi-stationary methods. For all data periods, the dynamic methods converge must faster to a reliable estimation result. Even more, the dynamic analysis methods result in accurate resistance estimates for summer data sets, while the semi-stationary methods do not 630 lead to reliable results. Next to that, the performance among

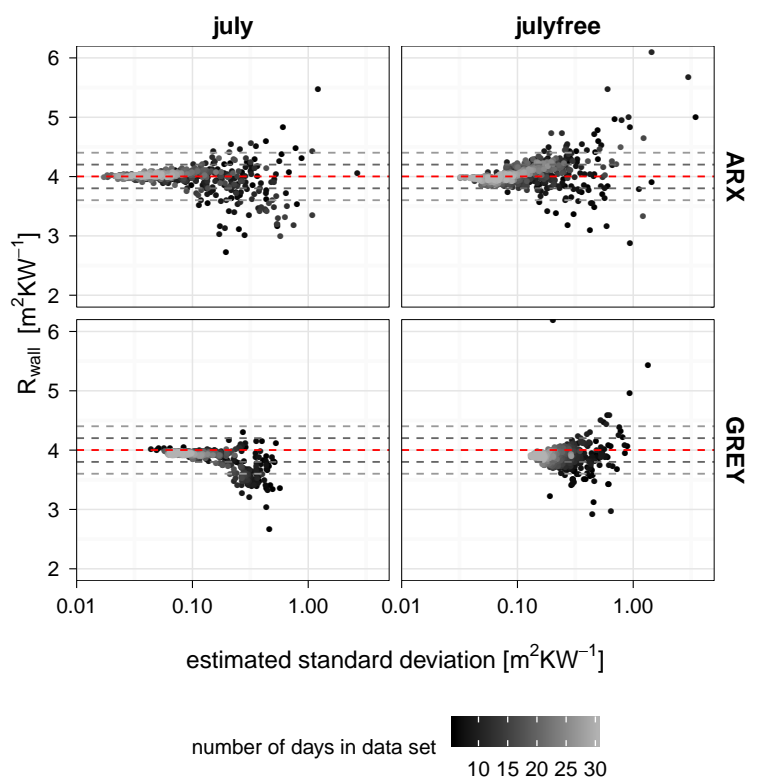

Figure 4: The thermal resistance estimates resulting from data sets larger than 5 days in function of their estimated standard deviation. quality. For the summer scenario with a semi-controlled indoor air temperature, Anderlind's regression method has a somewhat faster convergence to the goal value, whereas for the summer scenario with a free floating indoor air temperature, the greybox models are preferred. Nevertheless, for long enough data periods, the performance of the three methods is similar. The performance of the ARX-models might be more sensitive to particularities in some data sets. Nevertheless, these less reliable results are indicated by the estimated standard deviations accompanying the results.

\section{Application on experimental data}

\subsection{Case study}

In the previous section, the analysis methods were applied on numerically simulated data to be able to compare the results with the 'real' goal value and to neglect time dependent variations of the thermal resistance. In this section, the different analysis methods will be applied on actual measurement data of a south-facing insulated cavity wall. The measurements are performed in the VLIET test building of the KU Leuven, to study the hygrothermal behaviour of building components under real climatic conditions. Measurements of 12 cavity walls are available from experiments during the late nineties and have previously been used to investigate the performance of brick cavity walls in [1].

The wall considered in this paper is similar to the previously simulated wall and will be characterised for winter and summer measurements. The test wall consists of an inner and outer brick leave of $0.140 \mathrm{~m}$ and $0.090 \mathrm{~m}$ respectively and is insulated with $0.140 \mathrm{~m}$ glass wool blankets. The brick façade is of red 


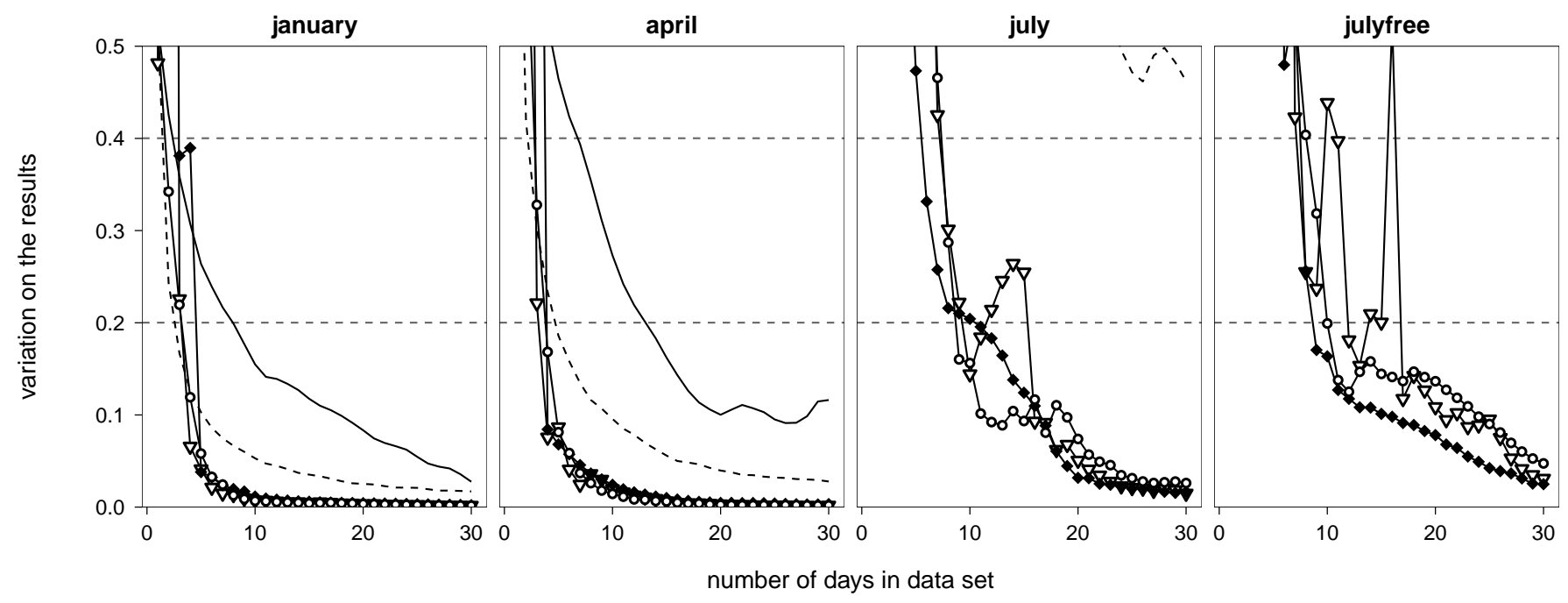

methods $\longrightarrow$ average method - - storage effects $\multimap-$ Anderlind $\rightarrow$ ARX $\multimap$ GREY

Figure 5: Comparison of the spread on the results as a function of the data set length for the different methods and different seasonal weather conditions. The grey dotted lines correspond to $5 \%$ and $10 \%$ deviations from the goal value.

Table 3: Thermal properties of the measured cavity wall from outside to inside.

\begin{tabular}{llll}
\hline & $\mathrm{d}$ & $\mathrm{R}$ winter / summer & $\mathrm{C}$ \\
& {$[\mathrm{m}]$} & {$\left[\mathrm{m}^{2} K W^{-1}\right]$} & {$\left[M J m^{-2} K^{-1}\right]$} \\
\hline brick & 0.090 & 0.112 & 0.171 \\
air cavity & 0.010 & 0.160 & $/$ \\
glass wool blankets & 0.140 & $4.025 / 3.917$ & 0.005 \\
brick & 0.140 & 0.274 & 0.217 \\
\hline TOTAL & 0.380 & $\mathbf{4 . 5 7 1} / \mathbf{4 . 4 6 3}$ & $\mathbf{0 . 3 9 2}$
\end{tabular}

colour, thus having a higher absorption coefficient than the previously simulated wall. The thermal properties of the wall materials are summarized in table 3 and are obtained from measurements or product information of the used materials. As the thermal conductivity of the insulation layer is known to be temperature dependent, a different insulation resistance is calculated for the two main periods that will be considered. Therefore, the overall insulation temperature is assumed to equal the mean of the two surface temperatures averaged over the considered periods. Hence, the theoretical total thermal resistance of the wall amounts to $4.571 \mathrm{~m}^{2} . K . W^{-1}$ for winter periods and to $4.463 \mathrm{~m}^{2} . K . \mathrm{W}^{-1}$ for summer periods. These values are used as reference values, although they do not pretend to embody the actual as-built thermal resistance of the wo wall.

The test wall is situated in the south façade of the building and is subjected to the outdoor climate and to a constant indoor 680 environment. The latter is heated to a set temperature of $23^{\circ} \mathrm{C}$, but no cooling is applied in the building. Hence, during warmer periods in summer, the indoor air temperature can exceed the set temperature. This heating regime corresponds most to the regime of the simulations with a semi-controlled indoor air ${ }_{685}$ temperature. The measurements, i.e. the internal heat flux and

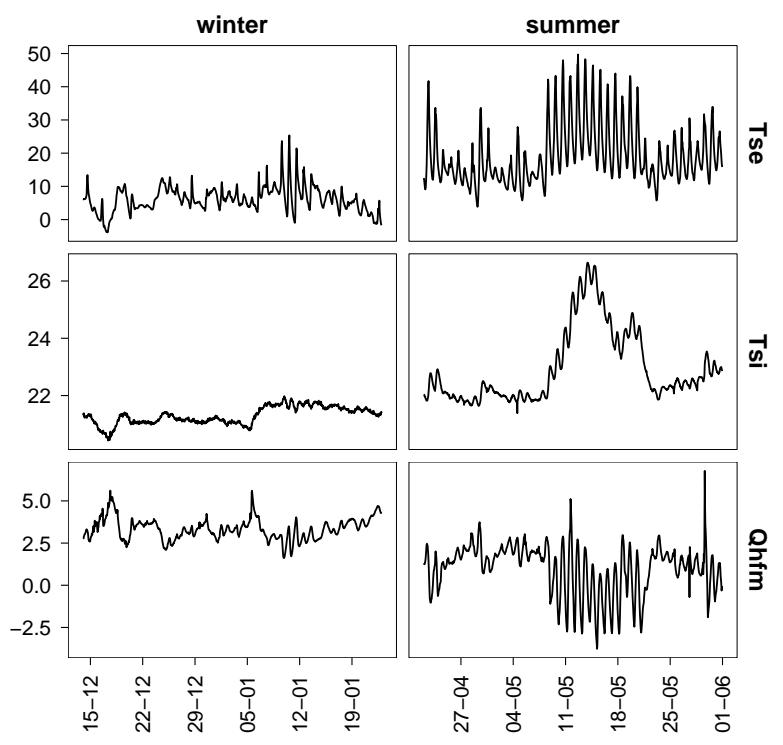

Figure 6: Experimental measurement data for the different seasonal periods with $T_{s e}$ the external surface temperature $\left({ }^{\circ} \mathrm{C}\right), T_{s i}$ the internal surface temperature $\left({ }^{\circ} \mathrm{C}\right)$ and $Q_{h f m}$ the heat flux $\left(W \cdot m^{-2}\right)$.

the internal and external surface temperatures of the wall, are recorded with a five minute interval but are averaged to four hourly values for the data analyses.

Two main data sets of 40 days are selected: one in winter from 14/12/1997 till 23/01/1998 and one in summer from $22 / 04 / 1998$ till 01/06/1998. The measurement data is represented in figure 6. Similar as to the simulation research, data sets with different lengths ranging from 1 to 30 days are 
constructed from these two main data periods using a moving window. However, the measurement data is now limited to 40 days in total and consequently, less larger data sets can be ${ }_{745}$ constructed compared to the simulation analysis. For example, only 10 data sets of 30 days will be analysed in winter, notably, the data set starting on the 14th of January till the data set starting on the 24 th of January.

\subsection{Results}

The estimation results of the thermal resistance of the test wall obtained by the different analysis methods are represented in figure 7. This figure is constructed analogously to figure 3:755 each data point represents an R-estimate resulting from a different data set. The results are plotted as a function of the length of the data sets and are ordered column wise according to the different seasonal periods, here winter and summer. The results for the different analysis methods are ordered row wise.

By examining the results of the data sets during winter periods, it is seen from figure 7 that all analysis methods lead to reliable R-estimates for the cavity wall. The only difference with the simulation results is that no significant improvement of the dynamic analysis methods compared to the average method 765 is seen for winter data sets. Only for data sets containing up to four days, the spread on the results of the average method is higher, although the differences are negligible. In general, it can be stated that all analysis methods perform equally well for winter data sets.

The estimates resulting from the summer data sets, however, more differences compared to the simulation results. Note that the indoor conditions of the test wall in summer resemble most the simulated summer scenario with a semi-controlled indoor air temperature. Only, for the actual measurements, the period during which free floating air temperatures occur is much longer than in the simulations.

The average method shows a wide spread of resulting Restimates for summer data sets, as can be seen in figure 7 . Unreliable estimation results are obtained for data sets up till 20 days, yet, for data sets containing more than 20 days, a conBased on the results from the simulation assessment, it is questioned whether this convergence is misleading and may be due to the fact that only a limited amount of longer data sets are considered. More specifically, a limited amount of data sets information.

When the correction for storage effects is applied for the summer estimations, no significant improvement of the estimated thermal resistances is found. Already in the simulation sestima-7ont, the correction had a limited impact on the estima tion from summer measurements. Now, in addition, the real thermal properties of the wall are not exactly known, as was the case for the simulation assessment. Hence, assumptions had to be made in order to apply the correction for storage effects.

740 Nevertheless, the assumptions that are made are well founded 795 and are based on detailed construction information of the test wall, measurements and manufacturing specifications.
The R-estimates for summer data sets resulting from Anderlind's regression method and ARX-modelling are very similar to each other. A solid convergence of the estimator is reached for all long data periods. However, for data sets containing less than 20 days a lot of outlier results are observed. These outliers seem to correspond to data sets with a mainly free floating indoor air temperature. Apparently, the presence of large parts of free floating data in the measurements jeopardizes the estimation results. Following from the simulation research, long enough periods of purely free floating data should allow a proper R-estimation. Also, data sets with changing heating strategies due to an only partly controlled indoor air temperature did not set a problem in the simulation research. However, the simulated data with the semi-controlled temperature scenario only contained a free floating period of five days. The difficulties caused by this fraction of the data could easily be hidden by the unreliabilities linked to shorter data sets. Nevertheless, when long enough data periods are considered, both the ARX-modelling approach and Anderlind's technique are able to reach accurate and reliable estimation results for the experimental data.

From figure 7 , it is seen that the results of the stochastic grey-box modelling approach are less sensitive to the changing heating strategies of the summer measurements: a slow but firm convergence is noticed. Note however, that the resulting estimate is slightly lower than the long-term estimates of the other characterisation methods.

\section{Discussion}

From both the simulation and experimental assessment, it was found that the stochastic grey-box modelling approach results in slightly lower R-estimates than the other dynamic analysis methods. A possible explanation lies in the reduced order of the grey-box models compared to the ARX-models. The grey-box models are of third order while the ARX-models can have a maximum order up to 18 (the maximum model order corresponds to three days of historical data with a sample time of four hours). Hence, the number of time constants that can be modelled is large for the ARX-models and limited to three for the stochastic grey-box models. The reduced order of the greybox models might result in small variations that are discarded to the noise models, without disturbing the white noise behaviour, rather than that they are contributing to the model behaviour. Increasing the grey-box model order, however, would lead to unidentifiabilities hindering the estimation procedure.

Up to this point, the methods are only assessed based on their estimation performance. However, their application complexity should also be discussed. For the considered methods, the semistationary methods are the most straight-forward to apply, as they rely on simple equations that do not involve time-intensive computations. Anderlind's regression method is also easy to apply, although decisions have to be made on the fraction of data that will be used as measurement data from the past. In comparison, the application of ARX-modelling requires more theoretical knowledge of time series analysis in order to deduce the gain of the models. Nevertheless, the implementation 


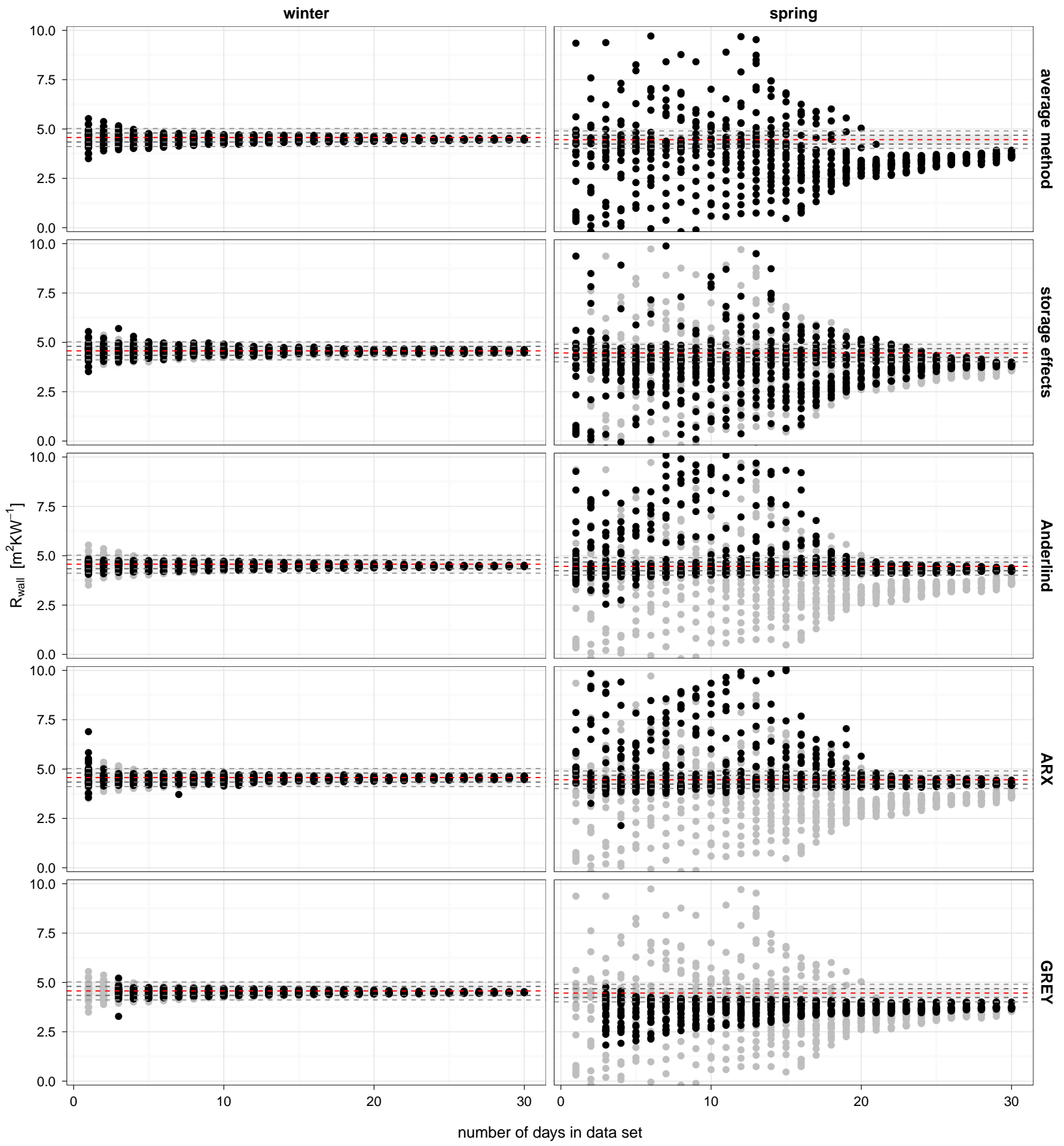

Figure 7: Comparison of the different analysis techniques regarding the data set length and period. The red dotted line represents the reference value for the thermal resistance of the cavity wall. The grey dotted lines and areas correspond to the $5 \%$ and $10 \%$ accuracy bands.

is based on regression techniques which can be computed by most elementary numerical software packages. Only if an automatic model order selection is applied, more advanced soft-805 ware is required. Finally, grey-box modelling is the most complex method to apply. The method is rooted in the fundamental stochastic framework of system identification and requires a decent understanding of the basics in order to perform a solid parameter estimation. However, despite the increased application efforts, the method offers a large variety of modelling possibilities and an elaborate framework of model validation tools in 

components from on-site measurements. Energy and Buildings 130: 309-320. doi.org/10.1016/j.enbuild.2016.08.061

return.

\section{Conclusions}

In this paper, a large-scale comparison of the available characterisation methods for building components in-situ is made. Thereby, special attention is given to the reliability of the meth- ${ }^{865}$ ods estimation results when confronted with data sets of limited measurement time spans and different seasonal boundary conditions. First, the methods' performance is assessed for simulated measurements of a south-facing insulated cavity wall in a moderate European climate. Subsequently, the performance is examined for actual measurement data of a similar test wall. The case study of a cavity wall facing south is deliberately choen because the methods' limitations are more pronounced for capacitive building components.

From the simulation research, it followed that the dynamic data analysis methods have an improved performance compared to the semi-stationary methods. By modelling the variations of the heat flux rather than cancelling them out, the dynamic methods are able to cope with the influences from the weather conditions in a better way. Even more, they are able to $_{885}$ deduce reliable thermal resistance estimates from typical summer measurements, which are characterised by a large capaciare not.

Among the different dynamic methods, no real preference is shown for a particular method. From the comparative assessment, it is seen that Anderlind's approach has a slightly faster convergence to accurate estimates, while the ARX-models and ${ }_{895}$ grey-box models have a slightly more accurate convergence, but for longer data periods. From a time management related point of view, stochastic grey-box modelling is more labourintensive than Anderlind's regression and ARX-modelling tech- ${ }_{900}$ niques. However, grey-box modelling enables the use of a set of validation tools that is not included in the other methods.

From the experimental research, it was concluded that the semi-stationary methods perform equally well as the dynamic $c_{905}$ analysis methods when winter data sets are considered. In contrast, for spring and summer data sets, only the dynamic methods lead to reliable estimation results. Nevertheless, for summer periods, it was seen that indoor boundary conditions chang-910 ing between controlled and free-floating indoor air temperature regimes hindered the estimation procedure. Only for sufficiently long data periods these difficulties are over mounted.

Generally, the semi-stationary methods are easy-to-use and ${ }_{915}$ are reliable characterisation methods when they are applied for winter measurements, whereas the dynamic methods are more complex in use, but offer a more versatile applicability.

\section{Acknowledgments}

Research funded by a Ph.D. grant (grant number 121167) of the Agency for Innovation by Science and Technology (IWT).

\section{References}

[1] H. Hens, A. Janssens, W. Depraetere, J. Carmeliet, J. Lecompte, Brick Cavity Walls: A Performance Analysis Based on Measurements and Simulations, Journal of Building Physics 31 (2) (2007) 95-124. doi:10.1177/1744259107082685.

URL http://jen.sagepub.com/cgi/doi/10.1177/1744259107082685

[2] R. Lowe, J. Wingfield, M. Bell, J. Bell, Evidence for heat losses via party wall cavities in masonry construction, Building Service Engineering Research and Technology 28 (2) (2007) 161-181. doi: $10.1177 / 0143624407077196$.

URL http://bse. sagepub.com/cgi/doi/10.1177/0143624407077196

[3] S. Flanders, Confidence in Heat Flux Transducer Measurements of Buildings, in: ASHRAE Transactions, Vol. 91, Chicago, 1985.

[4] J. Fang, R. Grot, In Situ Measurement of the Thermal Resistance of Building Envelopes of Office Buildings, in: ASHRAE Transactions, Vol. 91, Chicago, 1985, pp. 543-557.

[5] ISO 9869: 2014 Thermal insulation - Building elements - In-situ measurement of thermal resistance and thermal transmittance (2014).

[6] I. Naveros, M. Jiménez, M. Heras, Analysis of capabilities and limitations of the regression method based in averages, applied to the estimation of the $\mathrm{U}$ value of building component tested in Mediterranean weather, Energy and Buildings 55 (2012) 854-872. doi:10.1016/j.enbuild.2012.09.028.

URL http://dx.doi.org/10.1016/j.enbuild.2012.09.028 http://linkinghub.elsevier.com/retrieve/pii/S0378778812004811

[7] A.-H. Deconinck, S. Roels, Comparative assessment of in - situ thermal characterisation, in: J. Arfvidsson, L.-E. Harderup, A. Kumlin, B. Rosencrantz (Eds.), Proceedings of the 10th Nordic Symposium on Building Physics, 10th Nordic Symposium on Building Physics, Lund, Sweden, 2014, pp. 525-532.

[8] A. Aittomäki, Determination of the Overall Heat Transfer Coefficient of Multilayer Structures under Non-Steady Conditions, CIB W 40, Holzkirchen-meeting 18-20 Sept.

[9] C. Kupke, Unteruchungen über ein Wärmedämm - Schnellmesverfahren, Institut für Bauphysik, Stuttgart, BW 148/76.

[10] S. Ahvenainen, E. Kokko, A. Aittomäki, Thermal Conductance of Wall Structures, LVI-Tekniikan Laboratorio, Report 54, Espoo.

[11] I. Roulet, C. Gass, J. Markus, In-Situ U-Value Measurement : Reliable Results in Shorter Time By Dynamic Interpretation of Measured Data, in: Buildings III Conference, 1985.

URL http://web.ornl.gov/sci/buildings/2016/1985 B3 papers $/ 057 . p d f$

[12] G. Anderlind, Dynamic thermal models. Two dynamic models for estimating thermal resistance and heat capacity from in situ measurements., Tech. rep., The Swedish Council for Building Research, Stockholm (1996).

URL http://gadbyggnadsfysik.se/data/documents/BFR-Dynamic-Therm

[13] G. Anderlind, Multiple Regression Analysis of in situ Thermal Measurements - Study of an Attic Insulated with $800 \mathrm{~mm}$ Loose Fill Insulation, Journal of Building Physics 16 (1) (1992) 81-104. doi:10.1177/109719639201600109.

URL http://jen. sagepub.com/cgi/doi/10.1177/109719639201600109

[14] U. Norlén, Determining the Thermal Resistance from In-Situ Measurements, in: J. Bloem (Ed.), Workshop on Application of System Identification in Energy Savings in Buildings, commission of the european communities, 1994, pp. 402-429.

[15] U. Norlén, Estimating thermal parameters of outdoor test cells, Building and Environment 25 (1) (1990) 17-24. doi:10.1016/03601323(90)90036-Q.

URL http://linkinghub.elsevier.com/retrieve/pii/036013239090036

[16] M. Jiménez, H. Madsen, K. Andersen, Identification of the main thermal characteristics of building components using MATLAB, Building and Environment 43 (2) (2008) 170-180. doi:10.1016/j.buildenv.2006.10.030. URL http: //linkinghub.elsevier.com/retrieve/pii/S03601323060029

[17] I. Naveros, C. Ghiaus, D. Ruíz, S. Castaño, Physical parameters identification of walls using ARX models obtained by deduction, Energy and Buildings 108 (2015) 317-329. doi:10.1016/j.enbuild.2015.09.021. URL http://linkinghub.elsevier.com/retrieve/pii/S03787788153026

[18] P. Biddulph, V. Gori, C. a. Elwell, C. Scott, C. Rye, R. Lowe, T. Oreszczyn, Inferring the thermal resistance and effective thermal mass 
of a wall using frequent temperature and heat flux measurements, Energy and Buildings 78 (2014) 10-16. doi:10.1016/j.enbuild.2014.04.004.

[19] P. Baker, H. van Dijk, PASLINK and dynamic outdoor testing of building components, Building and Environment 43 (2) (2008) 143-151. doi:10.1016/j.buildenv.2006.10.009.

URL http://linkinghub.elsevier. com/retrieve/pii/S0360132306002940

[20] O. Gutschker, Parameter identification with the software package LORD, Building and Environment 43 (2) (2008) 163-169. doi:10.1016/j.buildenv.2006.10.010.

URL http://linkinghub.elsevier.com/retrieve/pii/S0360132306002964

[21] M. J. Jiménez, B. Porcar, M. R. Heras, Application of different dynamic analysis approaches to the estimation of the building component U value, Building and Environment 44 (2) (2009) 361-367. doi:10.1016/j.buildenv.2008.03.010.

[22] F. G. N. Li, A. Smith, P. Biddulph, I. G. Hamilton, R. Lowe, A. Mavrogianni, E. Oikonomou, R. Raslan, S. Stamp, A. Stone, A. Summerfield, D. Veitch, V. Gori, T. Oreszczyn, Solid-wall U -values: heat flux measurements compared with standard assumptions, Building Research \& Information 43 (2) (2015) 238-252. doi:10.1080/09613218.2014.967977. URL http: //www . tandf online.com/doi/abs/10.1080/09613218.2014.967977

[23] I. Naveros, P. Bacher, D. P. Ruiz, M. J. Jiménez, H. Madsen, Setting up and validating a complex model for a simple homogeneous wall, Energy and Buildings 70 (2014) 303-317. doi:10.1016/j.enbuild.2013.11.076.

URL http: //linkinghub.elsevier.com/retrieve/pii/S0378778813007937 http://dx.doi.org/10.1016/j.enbuild.2013.11.076

[24] A.-H. Deconinck, S. Roels, A Maximum Likelihood Estimation of the Thermal Resistance of a Cavity Wall from On-site Measurements, Energy Procedia 78 (2015) 3276-3281. doi:10.1016/j.egypro.2015.11.723.

URL http://linkinghub.elsevier.com/retrieve/pii/S1876610215024558

[25] N. R. Kristensen, H. Madsen, S. B. Jørgensen, A method for systematic improvement of stochastic grey-box models, Computers \& Chemical Engineering 28 (8) (2004) 1431-1449. doi:10.1016/j.compchemeng.2003.10.003.

URL http://linkinghub.elsevier.com/retrieve/pii/S0098135403002758

[26] N. R. Kristensen, H. Madsen, S. B. Jørgensen, Parameter estimation in stochastic grey-box models, Automatica 40 (2) (2004) 225-237. doi:10.1016/j.automatica.2003.10.001.

URL http://linkinghub.elsevier.com/retrieve/pii/S000510980300298X

[27] M. Jiménez, B. Porcar, M. Heras, Estimation of building component UA and gA from outdoor tests in warm and moderate weather conditions, Solar Energy 82 (7) (2008) 573-587. doi:10.1016/j.solener.2008.02.013. URL http://linkinghub.elsevier. com/retrieve/pii/S0038092X08000327

[28] H. Janssen, B. Blocken, J. Carmeliet, Conservative modelling of the moisture and heat transfer in building components under atmospheric excitation, International Journal of Heat and Mass Transfer 50 (5-6) (2007) 1128-1140. doi:10.1016/j.ijheatmasstransfer.2006.06.048.

URL http://linkinghub.elsevier.com/retrieve/pii/S0017931006005011

[29] S. Sharples, Full-scale measurements of convective energy losses from exterior building surfaces, Building and Environment 19 (1) (1984) 31-39. doi:10.1016/0360-1323(84)90011-8.

URL http: //linkinghub.elsevier.com/retrieve/pii/0360132384900118

[30] L. Ljung, System Identification: Theory for the User, 2nd Edition, Prentice Hall PTR, Upper Saddle River, NJ, USA, 1999.

[31] H. Madsen, P. Bacher, G. Bauwens, A.-H. Deconinck, G. Reynders, S. Roels, E. Himpe, G. Lethé, Thermal Performance Characterization using Time Series Data - IEA EBC Annex 58 Guidelines, Technical University of Denmark (DTU), 2015.

985 [32] A. Janssens, S. Roels, L. Vandaele, Full scale test facilities for evaluation of energy and hygrothermal performances, Ugent. BFG, 2011. 